\title{
HIV Extracellular Tat: Myth or Reality?
}

\author{
Erwann Loret* \\ ETRAV laboratory (Aix Marseille University/CNRS), Faculty of Pharmacy, 27 Boulevard Jean Moulin, 13385 \\ Marseille, France
}

\begin{abstract}
The human immunodeficiency virus type 1 (HIV) eradication will require elimination of HIV infected cells. No antiretroviral treatments (ART) or vaccine approaches have been able to reduce significantly the level of HIV infected cells in peripheral blood. This inefficacy is generally explained by the presence of a major reservoir of latent HIV infected cells in the central nervous system (CNS) that would be a sanctuary where Cytotoxic T Lymphocytes (CTL) have no access and would refresh peripheral blood with activated HIV infected cells. In this review, the presence of a major reservoir in the CNS appears to be inconsistent with recent clinical studies measuring HIV DNA. The major reservoirs are gut tissue, rectal tissue and the peripheral blood where HIV infected cells survive in an environment containing CTL. Extracellular Tat might protect HIV infected cells from CTL due to its capacity to cross CTL membranes and trigger apoptosis. Evidences of Tat secretion from HIV infected cells are shown with the detection of Tat antibodies in different clinical studies. Presence of neutralizing Tat antibodies in cohorts of patients who were exposed to HIV but who are now seronegative is described. The conclusion of this review is that a vaccine eliciting neutralizing antibodies against Tat might significantly reduce the level of HIV infected cells, what ART or other vaccine approaches have been unable to achieve now. It could be a first step towards HIV eradication.
\end{abstract}

Keywords: HIV, HIV DNA, reservoirs, sanctuary, Tat, vaccine.

\section{INTRODUCTION}

A major obstacle in HIV eradication is the ability of the virus to remain in a subpopulation of cells it infects [1]. ART in blocking the HIV cycle at different levels quickly suppress HIV replication in patients and the viral particles load (viraemia) become no longer detectable in the peripheral blood [2]. ART (or cART) are different combinations of three to four antiviral molecules (from a pool of more than twenty molecules available now) that block HIV cycle at different levels and these combinations are also named HAART in the literature (Highly Active Anti-Retroviral Treatments) to recall that preliminary antiviral treatments before 1996 were unable to reduce viraemia to a long term non detectable level. However, even after years of ART, the treatment interruption results in the recurrence of viraemia in three weeks [3]. HIV infects mainly CD4 $+\mathrm{T}$ lymphocytes (CD4) and the reservoirs of HIV infected cells are made of latent CD4 cells [4]. The immune system needs to have latent CD4 cells with a long life to keep memory of former infections (latent memory CD4 cells are essential in the immune protection provided by a vaccine that can stand for the life of a human being) but activated CD4 cells have life expectancy of $24 \mathrm{~h}$ [2]. The latent HIV infected CD4 cells reservoir in peripheral blood is extremely stable and even in treated patients who have no detectable viraemia for seven years, the reservoir decays so slowly (50\% in 44 months) that eradication would require more than 70 years [5]. Although patients can stay for years with an undetectable viraemia in routine assays (less than

*Address correspondence to this author at the ETRAV laboratory (Aix Marseille University/CNRS), Faculty of Pharmacy, 27 Boulevard Jean Moulin, 13385 Marseille, France; E-mail: erwann.loret@univ-amu.fr
HIV RNA 40 copies per ml), ultrasensitive assays show that viraemia persists between 1 to 5 HIV RNA copies per ml [6]. Patients under ART remain seropositive to HIV GP 120 envelop protein due to these few viral particles still present in the peripheral blood.

The quest for a vaccine against AIDS has been a major issue for the scientific community since the cloning and the identification of HIV as the causative agent of AIDS [7-8]. Classical vaccination approaches were successful in the control of various viral diseases by priming or boosting the immune system to recognise viral envelope proteins and eradication of small pox in the world showed the efficacy of vaccination at a world scale [9]. However, vaccination fails when the antibody targets (epitopes) on viral envelop proteins change every year, such as influenza virus or even more rapidly for HIV [10]. Another difficulty, rarely evocated is that antibodies against HIV envelop proteins could participate to the progression to AIDS [11]. HIV envelop proteins in a complex with the CD4 membrane receptor might lead in a long term to an auto-immune response against CD4 cells and explain the decrease of CD4 cells, not related to HIV infection [12]. Consequently, vaccine trials targeting envelope proteins were stopped by the French AIDS Research National Agency (ANRS) that made the choice since 15 years to develop only vaccines eliciting a cellular response against HIV infected cells [13]. Cytotoxic T Lymphocytes (CTL) are certainly the immune cells most susceptible to eradicate HIV infected cells due to their immune specificity and ANRS has promoted four vaccine trials on HIV infected volunteers with multi-epitope vaccines called "lipopeptide" consisting of known CTL epitopes conserved across HIV subtypes, but these vaccines have so far fallen short of inducing robust and consistent anti-HIV 
CTL responses [14]. A new protocol with a mix of different lipopeptide vaccines is planned by ANRS on non HIV infected volunteers [15].

The inefficacy of ART or vaccines to decrease the level of HIV infected cells at least in the peripheral blood has been explained by the presence of a sanctuary for HIV infected cells, where CTL have no access [1]. Before 1996, the major reservoir of HIV infected cells was considered to be mainly the peripheral blood and the inefficacy of CTL to eliminate HIV cells was associated with the constant variability of HIV [2]. ART block the variability of HIV and HIV infected cells should have decreased or disappeared due to CTL but such was not the case [5]. Therefore, the hypothesis that the major reservoir of latently HIV infected cells could be in a sanctuary where CTL have no access became very attractive. This sanctuary, composed of latently HIV infected cells, would refresh permanently the peripheral blood with migration of activated HIV infected cells producing a basal level of viral particles. This sanctuary exists and it is the Central Nervous System (CNS) with its blood-brain barrier blocking CTL [1]. The problem is that CD4 cells, HIV infected or not, cannot cross the blood-brain barrier either. It was proposed that hematopoietic stem cells produced by bone marrow could be infected by HIV before migrating to CNS and differentiate in macrophages in a hypothesis referred to the "Trojan Horse" mechanism [1].

\section{CLINICAL STUDIES WITH HIV DNA MEASUREMENTS SHOW THAT THE CENTRAL NERVOUS SYSTEM (CNS) IS NOT THE MAJOR RESERVOIR OF HIV INFECTED CELLS}

The latently infected CD4 cell populations within a patient are low and location of the major reservoir of HIV infected cells in the CNS relies mainly on in vitro and in vivo experiments [4]. The development of HIV DNA measurement in the last ten years made it possible to locate and to quantify more precisely the different reservoirs since HIV infected cells contain a DNA form of HIV and virus particles contain an RNA form of HIV. Data are expressed differently with HIV RNA copies/ml of blood for viral particles and HIV DNA copies/million of Peripheral Blood Mononuclear Cells (PBMC) for HIV infected cells (HIV DNA is also expressed in $\log$ ). PBMC are hematopoietic stem cells that begin to differentiate in the bone morrow to become precursor of CD4 cells. PBMC can be infected by HIV before their differentiation in CD4 cell. Compared to the CNS, a tremendous higher level of HIV DNA was found in the Gut Associated Lymphoid Tissue (GALT) and the Rectal Associated Lymphoid Tissue (RALT) [16]. A high degree of sequence similarity between HIV in peripheral blood and HIV in GALT and RALT is compatible with a role of refreshment of HIV infected cells in peripheral blood [16]. Even with undetectable viraemia, patients have ongoing replication in these tissue reservoirs and there is an equilibrium with the peripheral blood compartment [17]. Other minor reservoirs of HIV infected cells were found in mesenteric lymph nodes, genital tract and intestinal macrophages [18].

HIV DNA is detected in patients only in the bone marrow cells containing PBMC and CD4 cells but HIV DNA cannot be found in purified CD34+ hematopoietic stem cells that can migrate to the CNS [19-20] and this is not in agreement with the "Trojan Horse" mechanism. CNS is certainly a minor reservoir of HIV infected cells, however, the cloning of HIV infected cells in the CNS revealed only non-viable HIV sequences and this is also not compatible with a reservoir supposed to continuously refresh the stock with viable activated HIV infected cells in the peripheral blood [21]. RALT and GALT that are obviously the major tissue reservoirs of HIV infected cells, have a fundamental difference with CNS they have a CD4/CD8 ratio equivalent to peripheral blood and therefore, cannot be considered as sanctuaries for HIV infected cells. Clinical studies also revealed that the level of HIV infected cells is higher than expected in the peripheral blood with HIV DNA ranging from 100000 copies/million PBMC in primo infection to 100 copies/million PBMC for patients under ART and less than 40 copies of HIV RNA per $\mathrm{ml}$ [22]. Although, the concentration of HIV DNA is twice higher in GALT and RALT, the main reservoir of HIV infected cells might be the peripheral blood due to its volume. Most importantly, the overwhelming majority of latent HIV infected CD4 cells in the peripheral blood, GALT or RALT survives in an environment with CTL and not in a sanctuary without CTL such as CNS.

\section{ROLE OF EXTRACELLULAR TAT}

How latently HIV infected cells can survive to CTL is not consensual and amazingly, the role that the extracellular HIV Tat protein might play to protect HIV infected cells from CTL is usually not mentioned [23-24]. Different lead compounds binding on Tat or blocking Tat were discovered but no one was developed to be tested in clinical trials [2527]. Tat was first described as a Trans Activator of Transcription of HIV genes [28]. Tat is a short protein from 86 to 101 amino acid residues that is rapidly produced following HIV infection [29]. HIV mRNAs have difficulties to elongate because a RNA hairpin called TAR, present at the beginning of all mRNA transcripts, makes a complex with cell proteins that blocks the RNA polymerase [29]. RNA polymerase can continue the mRNA elongation after binding of Tat on TAR [30]. The result of this binding is a different activation of HIV genes transcription compared to a classical activator that binds on non-coding DNA sequences. Tat has another peculiarity that no other HIV proteins have, that is to be secreted by HIV infected CD4 cells [31, 32]. ART do not block the secretion of Tat even for HIV-1 infected patients with an undetectable viraemia [33]. Evidences of Tat secretion from HIV infected cells are shown with the detection of Tat antibodies in clinical studies in China [34], France [35], Italy [36], in Netherland [37], Uganda [38] and USA [39]. A serological survey for one year showed that Tat antibodies can disappear and/or appear with blood samplings carried out every three months in the same patient [35]. This is amazing compared to the stable presence of antibodies against GP 120. Fig. (1) shows sequences of Tat variants representative of the five main HIV subtype that have the same trans-activation activity in spite of mutations up to $40 \%$. Human Tat antibodies recognised mainly variable regions and do not have a cross clades capacity [38]. 


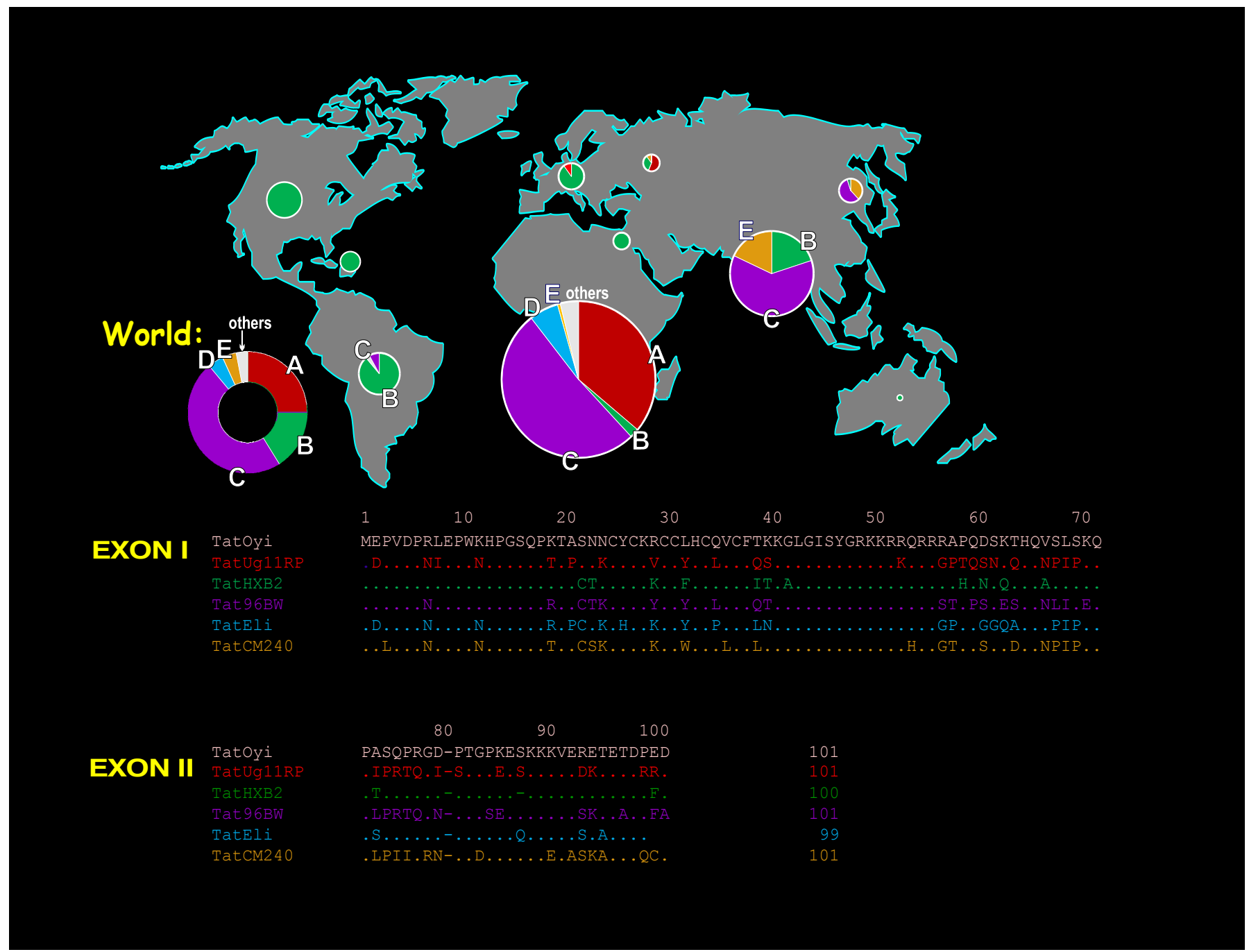

Fig. (1). There are five main HIV-1 subtypes in the world and Tat variability follows this geographical diversity of HIV with mutations up to $40 \%$ observed among Tat variants. These mutations do not modify Tat activity but affect Tat immune properties [29]. Tat Ug 11 RP, Tat HXB2, Tat 96BW, Tat Eli and Tat CM240 represent respectively HIV-1 subtypes A, B, C, D and E and are currently used to study the immune response against Tat in HIV infected patients [33] or in the Tat Oyi vaccine clinical trial (data not shown).

How the secretion of extracellular Tat might protect HIV infected cells from CTL is related to the amazing capacity of Tat to cross membranes [29]. Fig. (1) shows that there is a basic region made of Lys and Arg that is rather well conserved among Tat variants corresponding to residues (47)YGRKKRRQRR(57)R. This sequence is a "cargo" and can be attached to different proteins, DNA, RNA, or drugs to transport them across the cell membrane in an apparently energy-independent manner. How this highly hydrophilic peptide is able to cross the hydrophobic barrier imposed by the cell membrane is under investigation [40]. Apoptosis of CTL is observed in the presence of extracellular Tat in vitro [41]. The extracellular Tat crosses the membrane of Jurkaat cells (CTL cancer strain) to bind to the cytoskeleton proteins called tubulins, triggering what is called the "mitochondrial apoptosis" [29]. Nef is a HIV protein expressed at the same time with Tat and Nef blocks the mitochondrial apoptosis to prevent Tat mediated mitochondrial apoptosis in HIV infected CD4 cells [29]. A problem is that these in vitro experiments require $\mu$ molar concentrations of Tat that might be difficult to reach in vivo. Such a high concentration of extracellular Tat might be reached at proximity of CD4 infected cells due to the high level of Tat secretion [42]. Another explanation would be the occurrence of Tat non specific binding in vitro due to the basic region. The Tat basic region has similarities with a human protein called Protamine $\left({ }^{24}\right.$ RSCRRRKRRSCR $)$ that appears with sexual maturation. When Protamine is added to extracellular Tat in vitro, it significantly reduces the Tat concentration to induce Trans activation in Hela P4 cells or apoptosis in Jurkaat cells [29]. Therefore, extracellular Tat appears to be the best candidate to explain how latent HIV infected cells can survive in an environment containing CTL.

\section{CURATIVE TREATMENT AGAINST HIV NATURAL ACQUIRED RESISTANCE TO HIV}

AND

The only case of HIV eradication is a patient (called the Berlin patient) infected with HIV for more than 10 years, who had been diagnosed with acute myeloid leukemia. $\mathrm{He}$ received hematopoietic stem cells (HSC) transplantation from a donor who was homozygous, but had a specific 
mutation on a chemokine membrane receptor called delta 32 [43]. So far, the "Berlin patient" was the only successful case of HSC transplantation to eradicate HIV and other attempts failed. However, it shows that the first step of a curative treatment is a significant decrease of HIV infected CD4 cells in the peripheral blood and the second step is that the patient becomes seronegative [43]. It means that this patient has lost the capacity to produce antibodies against the HIV GP120 envelop protein because he has no more viral particles in his peripheral blood contrary to HIV infected patients under ART and an undetectable viraemia. Before the "Berlin patient" it was considered as impossible to be seropositive to GP 120 due to HIV infection and then to become seronegative.

The "Berlin patient" leads to reconsider patients Exposed and Persistently Seronegatives (EPS) to HIV. These patients have been neglected because they were considered as exposed, but were not HIV infected due to mutations, such as the delta 32 mutation. These patients are different from seropositive patients called Long Term Non Progressors (LTNP) who have a detectable viraemia, but succeed to maintain the level of their CD4 cells. The only well studied EPS cohort are Kenyan sex workers at Nairobi and they were considered as mutants for many years [44]. A major outbreak occurred 12 years ago in this cohort when one of these women became seropositive, while she was exposed to HIV for a decade. Ultrasensitive assays in the EPS Kenyan sex workers revealed the presence of HIV viral particles in tissues and now their resistance to HIV-1 appears to be related to their capacity to develop an efficient CTL response against HIV. However, CTL response in EPS Kenyan sex workers is five times lower in magnitude as compared to seropositive Kenyan sex workers who develop AIDS [45]. The former EPS Kenyan woman had probably lost naturally acquired immunity against HIV infection that she had for a decade.

Another example much less known is a study of a cohort of 25 seropositive women in Gabon in 1986 who became seronegatives $(n=23)$ after a survey of two years [46]. A HIV strain called HIV-1 Oyi was isolated from one of these women when she was still seropositive [46]. Contrary to the Kenyan sex workers who hade an activity that could induce a concentration of mutations protecting from HIV infection, the Gabonese women were identified from a pool of 750 seronegative pregnant women followed in the Franceville (Gabon) hospital in 1986 [46]. The high frequency of retro seroconversion in the Gabonese cohort suggested that a phenomenon of natural acquired immunity to HIV infection appeared in this cohort [29]. Focus was made on Tat Oyi that had mutations never observed in other Tat variants and Tat Oyi turned out to also have immune properties never observed in other Tat variants [29]. A Tat Oyi vaccine was tested on macaques with a heterologous mucosal SHIV challenge. SHIV infected CD4 cells were no longer detectable in the peripheral blood two months after SHIV challenge and one macaque became seronegative [29]. It was therefore possible to reproduce in vivo on the macaque model the same phenomenon of retro seroconversion observed in the Gabonese cohort. However, no study was made on the Gabonese cohort to know if they had antibodies against Tat and that cohort no longer exists since 1989. The only information obtained in 1999 was that the HIV infection markers no longer existed on these Gabonese women who were seropositive and had detectable viral particles in their peripheral blood in 1986 (Bernard Laporte, personal communication).

In collaboration with Gianfranco Pancino and Françoise Barre-Sinoussi 10 years ago, we had the opportunity to study the presence of Tat antibodies in sera of an EPS cohort of Vietnamese intravenous drug users (unpublished data). Patients in this cohort remained seronegative in spite of 15 years of drug use and sharing of HIV infected syringes but they were infected with other blood-borne viral infection [47]. We did an ELISA experiment with six Tat variants showed in Fig. (1), which is now a standard operating procedure in the lab. Currently, there are five main HIV-1 subtypes in the world: subtype A $(22 \%)$ is mainly in the East Africa; subtype B (10\%) is mainly in Europe and NorthAmerica; Subtype C (50\%) is predominant now in the world and is mainly in South Africa and India; subtype D (less than $5 \%$ ) is found mainly in Central Africa and subtype E (less than $5 \%$, now described as the recombinant form CRF_01AE) is found mainly in South East Asia [49]. Tat variability follows this geographical diversity of HIV with mutations up to $40 \%$ observed among Tat variants from $\mathrm{A}$, B, C, D and E HIV-1 subtypes, that do not modify Tat activity but affect Tat immune properties [29]. This is why we used Tat Ug $11 \mathrm{RP}$, Tat HXB2, Tat 96BW, Tat Eli and Tat CM240 to represent HIV-1 subtypes A, B, C, D and E (Fig. 1). These five synthetic proteins have a similar trans activation activity on HeLa P4 cells and a size going from 99 to 101 residues, which is now the dominant form of Tat in the field [29]. The Tat Oyi variant was added. We detected Tat antibodies in $66 \%$ of the sera from EPS patients $(n=25)$. No Tat antibodies were detected in sera from a cohort of HIV seronegative patients in France $(n=20)$. Tat antibodies were detected in $56 \%$ of the sera from a cohort of HIV seropositive patients in France $(n=27)$. The main difference between EPS Vietnamese and French HIV seropositive patients was the capacity for an EPS serum to have antibodies recognising different Tat variants, while French HIV seropositive patients were able to recognise mainly Tat HXB2. This result was obtained before the publication regarding the "Berlin Patient" and EPS Vietnamese were considered as exposed but non infected patients [48]. The presence of Tat antibodies revealed that at least two third of these EPS Vietnamese were HIV infected. The hypothesis is that EPS patients have neutralising antibodies against Tat variants that recognise a functional region highly conserved in all Tat variants. This is the rational of the Tat Oyi vaccination now in phase IIa at Marseille [50]. Other mechanisms of resistance to HIV infection were described for EPS Vietnamese who had no detectable antibodies against Tat [48].

Perhaps, the major EPS group who naturally resisted to HIV infection is new born babies from HIV infected mothers who escape from HIV infection without ART [51]. They have antibodies against GP120 until 12-14 months and then become seronegatives [52]. In the absence of ART, this retro seroconversion occurs for two thirds, born from HIV infected mothers. This high proportion excludes genetic factors that could provide an innate resistance against HIV. The prevalent hypothesis in the scientific community is that viral particles cannot cross the placenta barrier and 
the infection occurs for delivery, but it is also accepted that contamination can occur through breast feeding. However, a naturally acquired immunity was reported for a US baby girl called the 'Mississippi Baby' who had detectable HIV DNA in peripheral blood and got ART until she was 18 months old [53]. Then she became seronegative after HIV DNA was no longer detectable in her peripheral blood. She was declared cured from AIDS when she was 30 months old. She is four years old now, but unfortunately, HIV DNA was recently detected in her peripheral blood and she is no longer considered as cured. The "Mississipi baby" has certainly developed a natural immunity allowing eradication of HIV infected cells in her peripheral blood. The absence of viral particles in peripheral blood made her seronegative but, HIV infected cells were not eradicated from her tissue reservoirs. Probably, her natural immunity was an acquired phenomenon, which she lost resulting in the return of HIV infected cells in her peripheral blood.

\section{CONCLUSION}

Extracellular Tat might be the key target for a curative treatment against AIDS but an immune response against Tat appears not to stand for a long time contrary to the immune response against GP 120. Sequence analogy with human proteins such as Protamine may induce a repression of the immune system to recognize Tat particularly in adults. Eradication of HIV infected cells might be a long process in tissue reservoirs. A significant decrease of HIV DNA in the peripheral blood appears to be the first step for a curative treatment that ART or other vaccine approaches have been unable to achieve till now. Two phase II clinical trials are ongoing with Tat Oyi in France [50] and with a Tat variant closely related to Tat Oyi in South Africa [36]. If a significant decrease of HIV DNA in peripheral blood is observed in one or in these two trials, it will be a major advancement in HIV treatment comparable to ART in 1996.

\section{CONFLICT OF INTEREST}

The author confirms that this article content has no conflict of interest.

\section{ACKNOWLEDGEMENTS}

The author warmly thanks Isabelle Ravaux and Albert Darque (respectively main investigator and pharmacist of the EVATAT clinical trial) for their constant supports. The author thanks the staff of the Centre d'Investigation Clinique, Pharmacie à Usage Interne from the University Hospital Center "La Conception" and the Assistance Publique-Hôpitaux de Marseille for their commitment regarding the EVATAT clinical trial. The author thanks the students of Pharmacy and BTS for their help in the lab; and acknowledges Claire Cambo for reading of the MS. The author thanks Nicolas Castoldi and Younis Hermes from CNRS and Eric Berton from AMU for their support.

\section{REFERENCES}

[1] Alexaki A, Liu Y, Wigdahl B. Cellular Reservoirs of HIV-1 and their Role in Viral Persistence. Curr HIV Res 2008; 5: 388-400.
[2] Ho DD, Neumann AU, Perelson AS, et al. Rapid turnover of plasma virions and CD4 lymphocytes in HIV-1 infection. Nature 1995; 373: 123-6

[3] Chun TW, Davey RT Jr, Engel D., Lane HC, Fauci, AS (1999). Reemergence of HIV after stopping therapy. Nature 401; 10: 874-5.

[4] Chan CN, Dietrich I, Hosie MJ, Willet BJ. Recent developments in human immunodeficiency virus-1 latency research. J. Gen Virol 2013; 94: 917-32

[5] Siliciano JD, Kajdas J, Finzi D et al. Long-term follow-up studies confirm the stability of the latent reservoir for HIV-1 in resting CD4+ T cells. Nat Med 2003; 9: 727-8.

[6] Chun TW, Nickle DC, Justement JS, et al. Persistence of HIV in gut-associated lymphoid tissue despite long-term antiretroviral therapy. J Infect Dis 2008; 197:714-20.

[7] Barre-Sinoussi F, Chermann JC, Rey F et al. Isolation of a Tlymphotropic retrovirus from a patient at risk for acquired immune deficiency syndrome (AIDS). 1983. Science 1983; 220: 868-71.

[8] Gallo RC, Salahuddin SZ, Popovic M, et al. Frequent detection and isolation of cytopathic retroviruses (HTLV-III) from patients with AIDS and at risk for AIDS. Science 1984; 224: 500-3

[9] Smith KA. Edward Jenner and the Small Pox Vaccine. Front Immunol 2011; 14: 2-21.

[10] Pang S, Shlesinger Y, Daar ES, Moudgil T, Ho DD, Chen IS Rapid generation of sequence variation during primary HIV-1 infection. AIDS 1992; 5: 453-60.

[11] Cheingsong-Popov R, Panagiotidi C, Bowcock S, et al. Relation between humoral responses to HIV gag and env proteins at seroconversion and clinical outcome of HIV infection. BMJ 1991, 302: 23-6

[12] Caporossi AP, Bruno G, Salemi S, et al. Autoimmune T-cell response to the CD4 molecule in HIV-infected patients. Viral Immunol 1998; 11: 9-17.

[13] Levy JP Is AIDS vaccine research at a turning point? J. Hum Virol. 2000 3:83-87

[14] Sanou MP, De Groot AS, Murphey-Corb M, et al. HIV-1 Vaccine Trials: Evolving Concepts and Designs Open AIDS J 2012 6: 27488.

[15] Richert L, DoussauA, Lelièvre JD, et al. Accelerating clinical development of HIV vaccine strategies: methodological challenges and considerations in constructing an optimized multi-arm phase I/II trial design Trials 2014, 15: 68.

[16] Chun TW, Nickle DC, Justement JS, et al. Persistence of HIV in gut-associated lymphoid tissue despite long-term antiretroviral therapy. J Infect Dis 2008; 197: 714-20.

[17] Imamichi H, De Gray G, Dewar RL, et al. Lack of compartmentalization of HIV-1 quasi species between the gut and peripheral blood compartments. J Infect Dis 2011; 204: 309-14.

[18] Cory TJ, Schacker TW, Stevenson M, Fletcher CV. Overcoming pharmacologic sanctuaries. Curr Opin HIV AIDS 2013; 3: 190-5

[19] Josefsson L, Stockenstroma SV, Fariac NR, et al. The HIV-1 reservoir in eight patients on long-term suppressive antiretroviral therapy is stable with few genetic changes over time. PNAS 2013; 110: 4987-96.

[20] Durand CM, Ghiaur G, Siliciano JD, et al. HIV-1 DNA is detected in bone marrow populations containing CD4 $+\mathrm{T}$ cells but is not found in purified CD34+ hematopoietic progenitor cells in most patients on antiretroviral therapy. J Infect Dis 2012; 205: 1014-8.

[21] Gray LR, Cowley D, Crespan E, et al. Reduced basal transcriptional activity of central nervous system-derived HIV type 1 long terminal repeats. AIDS Res Hum Retroviruses 2013; 2: 365270.

[22] Bacchus C, Cheret A, Avettand F, et al. A single HIV-1 cluster and a skewed immune homeostasis drive the early spread of HIV among resting $\mathrm{CD} 4+$ cell subsets within one month post-infection. PLos One 2013; 8: e64219

[23] Katlama C, Deeks SG, Autran B, et al. Barriers to a cure for HIV: new ways to target and eradicate HIV-1 reservoirs. Lancet 2013; 381: 2109-17.

[24] Zolla-Pazner S A critical question for HIV vaccine development: which antibodies to induce? Science 2014; 6193: 167-8.

[25] Esquieu D., Péloponèse JM, Opi S et al. Discovery of a Tat HIV-1 inhibitor Through Computer-Aided Drug Design. Spectroscopy 2003; 17, 639-45.

[26] Narayan V, Ravindra KC, Chiaro C, Cary D, Aggarwal BB, Henderson AJ, Prabhu KS. Celastrol inhibits Tat-mediated human 
immunodeficiency virus (HIV) transcription and replication. J Mol Biol 2011; 410: 972-83

[27] Mousseau G, Clementz MA, Bakeman WN, et al. An analog of the natural steroidal alkaloid cortistatin A potently suppresses Tatdependent HIV transcription. Cell Host Microbe 2012; 12, 97-108

[28] Fisher AG, Ratner L, Mitsuya H, et al. Infectious mutants of HTLV-III with changes in the 3 ' region and markedly reduced cytopathic effects. Science 1986, 233: 655-9.

[29] Campbell, GR, Loret EP What does the structure-function relationship of the HIV-1 Tat protein teach us about developing an AIDS vaccine? Retrovirology 2009; 6: 50-63.

[30] Loret EP, Georgel P, Johnson WC Jr, Ho PS. Circular dichroism and molecular modeling yield a structure for the complex of human immunodeficiency virus type 1 trans-activation response RNA and the binding region of Tat, the trans-acting transcriptional activator. Proc Natl Acad Sci USA 1992; 89: 9734-8.

[31] Ensoli B, Barillari G, Salahuddin SZ, Gallo RC, Wong-Staal F. Tat protein of HIV-1 stimulates growth of cells derived from Kaposi's sarcoma lesions of AIDS patients. Nature. 1990; 345: 84-6.

[32] Loret EP, Vives E, Ho PS, Rochat H, Van Rietschoten J, Johnson WC Jr. Activating region of HIV-1 Tat protein: vacuum UV circular dichroism and energy minimization. Biochemistry 1991; 30: 6013-23.

[33] Mediouni S, Darque A, Baillat G, et al. Antiretroviral therapy does not block the secretion of the human immunodeficiency virus Tat protein. Infect Disord Drug Targets 2012; 1: 81-6.

[34] Chen Q, Li L, Liao W, et al. Characterization of Tat antibody responses in Chinese individuals infected with HIV-1. PLoS One 2013; 8: e60825.

[35] Mediouni S, Baillat G, Darque A, Ravaux I, Loret EP HIV-1 infected patients have antibodies recognizing folded Tat. Infect Disord Drug Targets 2011; 11: 57-63.

[36] Bellino S, Tripiciano A, Picconi O, et al. The presence of anti-Tat antibodies in HIV-infected individuals is associated with containment of CD4+ T-cell decay and viral load, and with delay of disease progression: results of a 3-years cohort study. Retrovirology 2014; 24: 11-49.

[37] van Baalen CA, Pontesilli O, Huisman RC, et al. Human immunodeficiency virus type 1 Rev- and Tat-specific cytotoxic $\mathrm{T}$ lymphocyte frequencies inversely correlate with rapid progression to AIDS. J Gen Virol 1997; 78: 1913-8

[38] Campbell GR, Senkaali D, Watkins J, et al. Tat mutations in an African cohort that do not prevent transactivation but change its immunogenic properties. Vaccine 2007; 25: 8441-7.
[39] Bachani M, Sacktor N, McArthur JC, Nath A, Rumbaugh J. Detection of anti-tat antibodies in CSF of individuals with HIVassociated neurocognitive disorders. J Neurovirol 2013; 19: 82-8.

[40] Herce HD, Garcia AE. Molecular dynamics simulations suggest mechanism for translocation of the HIV-1 TAT peptide across lipid membranes. Proc Natl Acad Sci USA 2007; 104: 20805-10

[41] Westendorp MO, Frank R, Ochsenbauer C, et al. Sensitization of T cells to CD95-mediated apoptosis by HIV-1 Tat and gp120. Nature 1995; 375: 497-500.

[42] Rayne F, Debaisieux S, Yezid H., et al. Phosphatidylinositol-(4,5)Bisphosphate enables efficient secretion of HIV-1 Tat by infected T-cells. EMBO J 2010; 8: 1348-62.

[43] Allers K, Hutter G, Hofmann J, et al. Evidence for the cure of HIV infection by CCR5Delta32/Delta32 stem cell transplantation. Blood 2011; 117: 2791-9.

[44] McMichael AJ, Rowland-Jones SL. Cellular immune responses to HIV. Nature 2001; 410: 980-7.

[45] Alimonti JB, Kimani J, Matu L, et al. Characterization of CD8 Tcell responses in HIV-1-exposed seronegative commercial sex workers from Nairobi, Kenya. Immunol Cell Biol 2006; 84: 482-5.

[46] Huet T, Dazza MC, Brun-Vezinet F, Roelants GE, Wain-Hobson S. A highly defective HIV-1 strain isolated from a healthy Gabonese individual presenting an atypical western blot. AIDS 1989, 3:70715

[47] Truong LX, Luong TT, Scott-Algara D, et al. CD4 cell and CD8 cell-mediated resistance to HIV-1 infection in exposed uninfected intravascular drug users in Vietnam. AIDS. 2003; 17: 1425-34.

[48] Saez-Cirion A, Versmisse P, Truong LX, et al. Persistent resistance to HIV-1 infection in CD4 $\mathrm{T}$ cells from exposed uninfected Vietnamese individuals is mediated by entry and post-entry blocks. Retrovirology 2006; 8: 81-9.

[49] Hemelaar J, Gouws E, Ghys PD, Osmanov S. Global and regional distribution of HIV-1 genetic subtypes and recombinants in 2004 AIDS 2006; 20: 13-23

[50] Mediouni S, Darque A, Ravaux I, et al. Identification of a highly conserved surface on Tat variants. J Biol Chem 2013; 288: 19072 80 .

[51] Rowland-Jones S, Pinheiro S, Kaul R. New insights into host factors in HIV-1 pathogenesis. Cell 2001; 104: 473-6.

[52] Beattie T, Rowland-Jones S, Kaul R. HIV-1 and AIDS: what are protective immune responses? J. VIH Ther 2002; 7: 35-9

[53] Persaud D, Gay H, Ziemniak C, et al. Absence of detectable HIV-1 viraemia after treatment cessation in an infant. $\mathrm{N}$ Engl $\mathrm{J}$ Med. $2013 ; 369,1828-35$. 